\title{
ERRATA FOR "CUBIC POLYNOMIAL MAPS WITH PERIODIC CRITICAL ORBIT, PART II: ESCAPE REGIONS"
}

\author{
ARACELI BONIFANT, JAN KIWI, AND JOHN MILNOR
}

\begin{abstract}
In this note we fill in some essential details which were missing from our paper. In the case of an escape region $\mathcal{E}_{h}$ with non-trivial kneading sequence, we prove that the canonical parameter $t$ can be expressed as a holomorphic function of the local parameter $\eta=a^{-1 / \mu}$ (where $a$ is the periodic critical point). Furthermore, we prove that for any escape region $\mathcal{E}_{h}$ of grid period $n \geq 2$, the winding number $\nu$ of $\mathcal{E}_{h}$ over the $t$-plane is greater or equal than the multiplicity $\mu$ of $\mathcal{E}_{h}$.
\end{abstract}

A result which can be stated as follows is claimed in $\S 6$ of the paper Cubic Polynomial Maps with Periodic Critical Orbit, Part II: Escape Regions, Conformal Geometry and Dynamics 14 (2010), 68-112 (referred to below as [BKM]).

Assertion A. For any escape region $\mathcal{E}_{h}$, the residue $\oint d t / 2 \pi i$ at the ideal point $\infty_{h}$ is zero. Furthermore, whenever the kneading sequence of $\mathcal{E}_{h}$ is non-trivial, the indefinite integral $t=\int d t$ can be expressed as a holomorphic function of the local parameter $\eta=\xi^{1 / \mu}=a^{-1 / \mu}$.

This assertion is true; however, there is a gap in our proof when the kneading sequence is non-trivial. In this case, [BKM, Lemma 5.19 and Theorem 6.2] do show that the quotient $d t / d a$ can be expressed as a locally holomorphic function of $\eta$, vanishing at $\eta=0$. However, this is not enough to prove the assertion 1 Since $a=\eta^{-\mu}$, we have

$$
\frac{d t}{d \eta}=\frac{d t}{d a} \frac{d a}{d \eta}=-\mu \frac{d t}{d a} \eta^{-\mu-1}
$$

Thus we must show that $d t / d a$ is divisible by $\eta^{\mu+1}$ in order to complete the proof. In fact, we will prove a slightly sharper statement. The necessary details follow.

Lemma B. Consider a Branner-Hubbard marked grid of period $2 n \geq 2$, denoting its finite column heights by $L_{1}, \ldots, L_{n-1}$. If $L_{n-1}>0$, then

$$
L_{j}=L_{n-1}-j \quad \text { for } \quad 1 \leq j \leq L_{n-1} .
$$

Received by the editors April 2, 2010.

2010 Mathematics Subject Classification. Primary 37F10, 30C10, and 30D05.

The first author was partially supported by the Simons Foundation.

The second author was supported by Research Network on Low Dimensional Dynamics PBCT/CONICYT, Chile.

${ }^{1}$ Our mistake was to ignore the $\xi^{2}$ in the denominator of BKM Equation (6.3)].

${ }^{2}$ The period $p$ of the critical orbit can be any multiple of the grid period $n$; but we will work only with the grid. Note that $n \geq 2$ if and only if the kneading sequence is non-trivial. 
Proof. Let $\left\{a_{i}\right\}$ be the periodic critical orbit. We will write the puzzle metric $d\left(a_{i}, a_{j}\right)$ of [BKM, Definition 3.7] briefly as $d(i, j)$, with $i, j \in \mathbb{Z} / n$, and with $d(0, i)=2^{-L_{i}}$. The argument will be based on the following statement from BKM. Lemma 3.8].

Expanding property. The equality

$$
d(i+1, j+1)=2 d(i, j)
$$

holds provided that $d(i, j)<1$, and provided that $\{0, i, j\}$ do not form the vertices of an equilateral triangle in this metric.

Using this, we will prove inductively that

$$
d(0, j)=d(j-1, j)=2^{j-N}
$$

for $1 \leq j \leq N$. To begin the induction, since the degenerate triangle with vertices $\{0,0, n-1\}$ is certainly not equilateral, the equation $d(0, n-1)=2^{-N}<1$ implies that

$$
d(1, n)=2 d(0, n-1)=2^{1-N} .
$$

Since $d(1, n)=d(0,1)$, this proves Equation $\left(*_{1}\right)$. Now suppose inductively that $\left(*_{j}\right)$ holds for $j<k$, where $2 \leq k \leq N$. Then the triangle $\{0, k-2, k-1\}$ is not equilateral, hence

$$
d(k-1, k)=2 d(k-2, k-1)=2^{k-N} .
$$

Together with the induction hypothesis, this proves that $d(0, k-1)<d(k-1, k)$. Therefore the ultrametric property (the statement that the two longest edges of any triangle must have equal length) implies that $d(0, k)=d(k-1, k)$. This completes the induction. Since $d(0, j)=2^{-L_{j}}$, we have also proved that $L_{j}=N-j$, as required.

It will be convenient to use the abbreviated notation $A_{\ell}(j)$ for the BrannerHubbard annulus $A_{\ell}\left(a_{j}\right)$. As in the proof of [BKM, Lemma 5.19], let3

$$
\mathfrak{S}_{j}=\sum_{\ell=0}^{\infty} \operatorname{MOD}\left(A_{\ell}(j)\right)
$$

be the sum of all of the moduli for the $j$-th column, normalized so that $\operatorname{MOD}\left(A_{0}(j)\right)=2$.

Lemma C. The inequality

$$
\mathfrak{S}_{1} \geq \mathfrak{S}_{n}+2=\mathfrak{S}_{0}+2
$$

holds whenever the grid period satisfies $n \geq 2$, with strict inequality when $n>2$.

Proof. As in the proof of the weaker inequality $\mathfrak{S}_{1}>\mathfrak{S}_{n}$ following the statement of [BKM], Lemma 5.19], the idea is to note that each critical modulus $\operatorname{MOD}\left(A_{\ell}(n)\right)$ is equal to some $\operatorname{MOD}\left(A_{\ell^{\prime}}(1)\right)$ from the first column, where the correspondence $\ell \mapsto \ell^{\prime}=\ell^{\prime}(\ell) \geq \ell$ is strictly monotone, with $\ell^{\prime}=\ell+n-1$ for large $\ell$.

\footnotetext{
${ }^{3}$ As an example, in Figures 1 and 2 , the moduli for the points in the zero-th column at depth $0 \leq \ell \leq 7$ can be computed from [BKM, Lemma 5.7] as 2, $1, \frac{1}{2}, \frac{1}{4}, 1, \frac{1}{2}, \frac{1}{4}$, $\frac{1}{8}$, with $\operatorname{MOD}\left(A_{\ell}(0)\right)=\operatorname{MOD}\left(A_{\ell-5}(0)\right) / 2$ for $\ell>7$. The sum is $\mathfrak{S}_{0}=\frac{31}{4}=7 \frac{3}{4}$.
} 


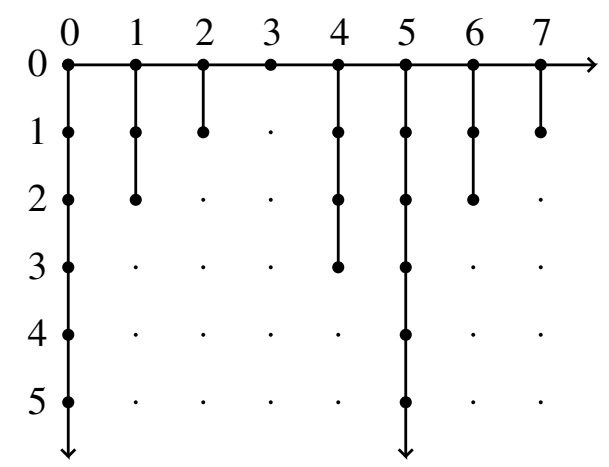

FIGURE 1. Sample grid of period $n=5$. Here the column heights are $L_{0}=\infty, L_{1}=2, L_{2}=1, L_{3}=0, L_{4}=3, \ldots$.

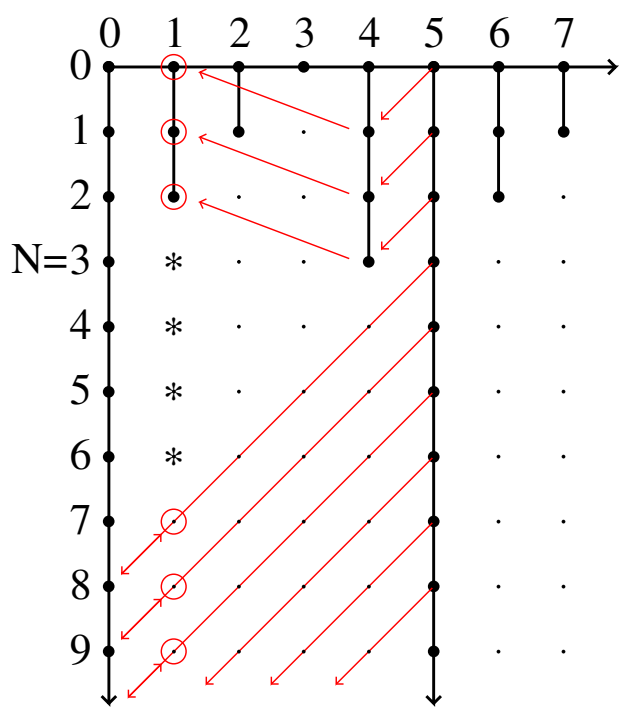

Figure 2. The correspondence $\ell \mapsto \ell^{\prime}$.

This correspondence can be described as follows. Start with the marked grid point in the $n$-th column at depth $\ell$ and follow the south-west diagonal until hitting another marked point, say in column $n-\delta$ at depth $\ell+\delta$. Then by definition

$$
\ell^{\prime}(\ell)=\ell+\delta-1
$$

one level higher than the hitting point. (Compare Figure 2, where each grid point of level $\ell^{\prime}$ in the first column is circled.) Using [BKM, Lemma 5.7], it is a straightforward exercise to prove that $\operatorname{MOD}\left(A_{\ell}(n)\right)$ is equal to $\operatorname{MOD}\left(A_{\ell^{\prime}}(1)\right)$. (Both are equal to $2 \operatorname{MOD}\left(A_{\ell^{\prime}+1}(0)\right)$.)

Evidently, there must be exactly $n-1$ levels which do not lie in the image of this correspondence $\ell \mapsto \ell^{\prime}$. The corresponding points in the first column are indicated by asterisks in Figure 2. Thus the difference $\mathfrak{S}_{1}-\mathfrak{S}_{n}$ is precisely equal to the sum of the $n-1$ moduli $\operatorname{MOD}\left(A_{\ell}(1)\right)$ associated with these asterisk points. Setting $N=L_{n-1} \geq 0$, it is easy to check that $\ell^{\prime}=\ell$ for $\ell<N$; but that $\ell^{\prime}>\ell$ when 
$\ell=N$. Thus the grid point at depth $N$ in column one will always be the highest asterisk point. Since it follows easily from Lemma B that $\operatorname{MOD}\left(A_{N}(1)\right)=2$, this proves Lemma C.

Proof of Assertion A. Setting $\delta=\mathfrak{S}_{1}-\mathfrak{S}_{n} \geq 2$, the proof of [BKM], Lemma 5.19 and Theorem 6.2] show that $d t / d a$ can be expressed as $\xi^{\delta}=\eta^{\delta \mu}$ multiplied by a function of $\eta$ which is holomorphic near the ideal point. Hence $d t / d \eta$ is equal to $\eta^{(\delta-1) \mu-1}$ multiplied by a locally holomorphic function. Since $\delta \geq 2$ and $\mu \geq 1$, we have $(\delta-1) \mu-1 \geq 0$. Therefore $d t / d \eta$ is locally holomorphic, which implies that the indefinite integral $t$ is locally holomorphic, as required.

In fact this argument proves a slightly stronger result. Choosing the additive constant so that $t$ vanishes at the ideal point, we see that $t$ is equal to $\eta^{(\delta-1) \mu}=\xi^{\delta-1}$ times a locally holomorphic function, where $\delta \geq 2$ with strict inequality when $n>2$. Setting

$$
t=\beta \xi^{\nu / \mu}+\text { (higher order terms) with } \quad \beta \in \mathbb{C}, \beta \neq 0,
$$

we obtain the following.

Assertion D. For any escape region of grid period $n \geq 2$, the winding number $\nu$ and the multiplicity $\mu \geq 1$ are related by the inequality $\nu \geq \mu$, with strict inequality when $n>2$.

\section{REFERENCES}

[BKM] A. Bonifant, J. Kiwi and J. Milnor, Cubic Polynomial Maps with Periodic Critical Orbit, Part II: Escape Regions, Conformal Geometry and Dynamics 14 (2010) 68-112.

Department of Mathematics, University of Rhode Island, 5 Lippitt Road, Room 200, Kingston, Rhode Island 02881

E-mail address: bonifant@math.uri.edu

Facultad de Matemáticas, Pontificia Universidad Católica, Casilla 306, Correo 22, Santiago de Chile, Chile

E-mail address: jkiwi@mat.puc.cl

Institute for Mathematical Sciences, Stony Brook University, Stony Brook, New YORK 11794-3660

E-mail address: jack@math.sunysb.edu 